\title{
A Variational Approach to an Inhomogeneous Second-Order Ordinary Differential System
}

\author{
B. Muatjetjeja and C. M. Khalique \\ International Institute for Symmetry Analysis and Mathematical Modelling, Department of Mathematical Sciences, \\ North-West University, Mafikeng Campus, Private Bag X2046, Mmabatho 2735, South Africa
}

Correspondence should be addressed to C. M. Khalique; masood.khalique@nwu.ac.za

Received 26 January 2013; Accepted 4 April 2013

Academic Editor: Teoman Özer

Copyright ( 2013 B. Muatjetjeja and C. M. Khalique. This is an open access article distributed under the Creative Commons Attribution License, which permits unrestricted use, distribution, and reproduction in any medium, provided the original work is properly cited.

This paper studies the coupled inhomogeneous Lane-Emden system from the Lagrangian formulation point of view. The existence of multiple positive solutions has been discussed in the literature. Here we aim to classify the system with respect to a first-order Lagrangian according to the Noether point symmetries it admits. We then obtain first integrals of the various cases which admit Noether point symmetries.

\section{Introduction}

The well-known coupled Lane-Emden systems

$$
\begin{gathered}
-\Delta u(x)=v^{p}(x), \quad x \in \Omega, \\
-\Delta v(x)=u^{q}(x), \quad x \in \Omega, \\
u(x), v(x)>0, \quad x \in \Omega, \\
u(x)=v(x)=0, \quad x \in \partial \Omega, \\
-\Delta u(x)=v^{p}(x)+\lambda f(x), \quad x \in \Omega, \\
-\Delta v(x)=u^{q}(x)+\lambda g(x), \quad x \in \Omega, \\
u(x), v(x)>0, \quad x \in \Omega, \\
u(x)=v(x)=0, \quad x \in \partial \Omega,
\end{gathered}
$$

where $\Omega$ is a domain in the $n$-dimensional Euclidean space $\mathbb{R}^{n}$ and $\Delta=\sum_{i=1}^{n}\left(\partial^{2} / \partial x_{i}^{2}\right)$ is the Laplacian on $\Omega$, arise naturally from the study of several physical phenomena such as pattern formation, population evolution, and chemical reactions (see [1] and references therein). These Lane-Emden systems (1)(2) have attracted considerable attention in recent years as can be seen from the number of papers published in the literature.
Several authors have studied the properties of solutions of (1), such as a priori estimates, existence and uniqueness results, Liouville-type theorems, and some symmetric and uniqueness results (see, e.g., [1-10]). For the inhomogeneous system (2), multiplicity results [11] have also been proved under certain conditions.

Recently, the authors of [12] studied the weakly coupled homogeneous second-order semilinear ordinary differential system

$$
\begin{gathered}
-u^{\prime \prime}(x)=v^{p}(x), \quad x \in(-l, l), \\
-v^{\prime \prime}(x)=u^{q}(x), \quad x \in(-l, l), \\
u(x), v(x)>0, \quad x \in(-l, l), \\
u(-l)=u(l)=v(-l)=v(l)=0,
\end{gathered}
$$

where $p, q>1$, and its corresponding inhomogeneous version

$$
\begin{gathered}
-u^{\prime \prime}(x)=v^{p}(x)+\lambda f(x), \quad x \in(-l, l), \\
-v^{\prime \prime}(x)=v^{q}(x)+\lambda g(x), \quad x \in(-l, l), \\
u(x), v(x)>0, \quad x \in(-l, l), \\
u(-l)=u(l)=v(-l)=v(l)=0 .
\end{gathered}
$$


It should be noted that the systems (3) and (4) are the onedimensional cases of the systems (1) and (2), respectively. A nondegeneracy result has been proved for positive solutions of the homogeneous system (3) and by making use of the nondegeneracy and uniqueness results of homogeneous system (3), sufficient and necessary conditions for the existence of multiple positive solutions for the inhomogeneous system (4) were obtained [12].

In this paper we study the coupled inhomogeneous LaneEmden system

$$
\begin{aligned}
& u^{\prime \prime}(t)+v^{p}(t)+\beta f(t)=0, \\
& v^{\prime \prime}(t)+u^{q}(t)+\beta g(t)=0,
\end{aligned}
$$

where $f$ and $g$ are arbitrary functions of $t$ and $p, q$ and $\beta$ are any arbitrary constants from the Lagrangian formulation point of view. We perform a Noether point symmetry classification of (5) with respect to a first-order Lagrangian. We then construct first integrals for the corresponding Noether operators. First integrals are of interest because they tell us something physical about the system [13] and can also be used to reduce the order of the differential equations.

\section{Fundamental Definitions about Noether Symmetries and First Integrals}

In this Section we recall some fundamental definitions about Noether point symmetries and first integrals which we utilize in Section 3.

We consider the vector field

$$
\Gamma=\tau(t, u, v) \frac{\partial}{\partial t}+\xi(t, u, v) \frac{\partial}{\partial u}+\eta(t, u, v) \frac{\partial}{\partial v},
$$

with first prolongation [14]

$$
\Gamma^{[1]}=\Gamma+(\dot{\xi}-\dot{u} \dot{\tau}) \frac{\partial}{\partial \dot{u}}+(\dot{\eta}-\dot{v} \dot{\tau}) \frac{\partial}{\partial \dot{v}},
$$

where $\dot{\tau}, \dot{\xi}$, and $\dot{\eta}$ denote total time derivatives of $\tau, \xi$, and $\eta$, respectively.

Definition 1. Consider the system of two second-order differential equations for the two unknowns $u$ and $v$, namely,

$$
\ddot{u}=\psi_{1}(t, u, v, \dot{u}, \dot{v}), \quad \ddot{v}=\psi_{2}(t, u, v, \dot{u}, \dot{v}) .
$$

A function $L(t, u, v, \dot{u}, \dot{v})$ is called a first-order Lagrangian of the system (8) if (8) are equivalent to the Euler-Lagrange equations $[15,16]$

$$
D_{t}\left(\frac{\partial L}{\partial \dot{u}}\right)-\frac{\partial L}{\partial u}=0, \quad D_{t}\left(\frac{\partial L}{\partial \dot{v}}\right)-\frac{\partial L}{\partial v}=0,
$$

where $D_{t}$ is the total differentiation operator defined by

$$
D_{t}=\frac{\partial}{\partial t}+\dot{u} \frac{\partial}{\partial u}+\dot{v} \frac{\partial}{\partial v}+\ddot{u} \frac{\partial}{\partial \dot{u}}+\ddot{v} \frac{\partial}{\partial \dot{v}}+\cdots .
$$

Definition 2. We call the vector field $\Gamma$ as in (6) a generator of a Noether point symmetry [15] associated with a first-order Lagrangian $L(t, u, v, \dot{u}, \dot{v})$ of the system (8) if there exists a potential function $B(t, u, v)$ such that

$$
\Gamma^{[1]}(L)+D_{t}(\tau) L=D_{t}(B) .
$$

The usefulness of an available Noether point symmetry is given in the following theorem.

Theorem 3 (Noether [17]). If $\Gamma$ of the form (6) is a Noether point symmetry generator corresponding to a first-order Lagrangian $L(t, u, v, \dot{u}, \dot{v})$ of the system (8), then

$$
I=B-\left[\tau L+(\xi-\tau \dot{u}) \frac{\partial L}{\partial \dot{u}}+(\eta-\tau \dot{v}) \frac{\partial L}{\partial \dot{v}}\right]
$$

is a Noether first integral of the system (8) associated with the operator $\Gamma$.

\section{Noether Symmetry Classification and the First Integrals for (5)}

To perform a Noether symmetry classification of the coupled inhomogeneous Lane-Emden system (5) it turns out that we need to consider four cases for different values of $p$ and $q$ separately.

3.1. $p \neq-1, q \neq-1$. In this case a first-order Lagrangian of the system (5) is given by

$$
L=\dot{u} \dot{v}-\frac{v^{p+1}}{p+1}-\frac{u^{q+1}}{q+1}-\beta(v f(t)+u g(t))
$$

The substitution of $L$ into (11) and separation with respect to powers of $\dot{u}$ and $\dot{v}$ yield linear overdetermined system of eight partial differential equations, namely,

$$
\begin{aligned}
& \tau_{u}=0, \\
& \tau_{v}=0, \\
& \xi_{u}+\eta_{v}-\tau_{t}=0, \\
& \xi_{v}=0, \\
& \eta_{u}=0, \\
& \eta_{t}=B_{u}, \\
& \xi_{t}=B_{v}, \\
&-\beta \tau v \dot{f}-\beta \tau u \dot{g}-\xi u^{q}-\beta g \xi \eta v^{p} \\
&-\beta f \eta-\tau_{t} \frac{v^{p+1}}{p+1}-\tau_{t} \frac{u^{q+1}}{q+1}-\beta v \tau_{t} f-\beta u \tau_{t} g=B_{t} .
\end{aligned}
$$


Solving the above system of equations, we obtain

$$
\begin{gathered}
\tau=a(t), \\
\xi=\frac{\dot{a} u}{2}-u h^{\prime}(v)+e(t), \\
\eta=\frac{\dot{a} v}{2}+\int b(t) d t+h(v), \\
B=\frac{\ddot{a}}{2} u v+b u+\dot{e} v+l(t), \\
\frac{1}{p+1}\left[\dot{a}+(p+1) \frac{\dot{a}}{2}\right] v^{p+1}-\frac{1}{q+1}\left[\dot{a}+(q+1) \frac{\dot{a}}{2}\right] u^{q+1} \\
+h^{\prime} u^{q+1}-\beta a(v \dot{f}+u \dot{g})-e u^{q} \\
-\beta g u\left(\frac{3 \dot{a}}{2}-h^{\prime}\right)+\beta g e-v^{p}\left(\int b d t+h\right) \\
-\frac{3}{2} \beta \dot{a} f v-\beta f\left(\int b d t+h\right)=\frac{1}{2} \ddot{a} u v+\dot{b} u+\ddot{e} v+\dot{l} .
\end{gathered}
$$

The analysis of (15) reveals the following three cases for which the Noether point symmetries exist.

Case 1. $f(t)=\delta, g(t)=\lambda$, where $\delta$ and $\lambda$ are constants.

In this case we obtain $\tau=c_{1}$, where $c_{1}$ is a constant, $\xi=0$, $\eta=0$, and $B=0$. Therefore we have a single generator of Noether symmetry, namely, $\Gamma=\partial / \partial t$. The use of the Noether integral (12) gives us the first integral

$$
I=\dot{u} \dot{v}+\frac{v^{p+1}}{p+1}+\frac{u^{q+1}}{q+1}+\beta(\delta v+\lambda u) .
$$

Case 2. $f(t)=\delta t^{-3 / 2}, g(t)=\lambda t^{-3 / 2}, p=-3$, and $q=-3$, where $\delta$ and $\lambda$ are constants.

In this case we obtain $\tau=2 t, \xi=u, \eta=v$, and $B=0$, and so we have a single Noether symmetry

$$
\Gamma=2 t \frac{\partial}{\partial t}+u \frac{\partial}{\partial u}+v \frac{\partial}{\partial v} .
$$

The formula (12) provides the first integral

$$
I=2 t \dot{u} \dot{v}-(u \dot{v}+\dot{u} v)+2 \beta t^{-1 / 2}(\delta v+\lambda u)-t\left(u^{-2}+v^{-2}\right) .
$$

Case 3. $f(t)=\delta t^{-3}, g(t)=\lambda t^{-3}, p=-3$, and $q=-3$, where $\delta$ and $\lambda$ are constants.

We get $\tau=t^{2}, \xi=u t, \eta=t v$, and $B=u v$, and so we have a single Noether symmetry generator

$$
\Gamma=t^{2} \frac{\partial}{\partial t}+u t \frac{\partial}{\partial u}+v t \frac{\partial}{\partial v} .
$$

The invocation of the Noether integral (12) results in the first integral

$$
\begin{aligned}
I= & t^{2} \dot{u} \dot{v}-t(u \dot{v}+\dot{u} v)+u v+\beta t^{-1}(\delta v+\lambda u) \\
& -\frac{t^{2}}{2}\left(u^{-2}+v^{-2}\right) .
\end{aligned}
$$

3.2. $p=-1, q=-1$. In this case the coupled inhomogeneous Lane-Emden system (5) becomes

$$
\begin{aligned}
& u^{\prime \prime}(t)+\frac{1}{v(t)}+\beta f(t)=0, \\
& v^{\prime \prime}(t)+\frac{1}{u(t)}+\beta g(t)=0,
\end{aligned}
$$

which has a Lagrangian

$$
L=\dot{u} \dot{v}-(\ln u v)-\beta(v f(t)+u g(t)) .
$$

Following the above procedure of Section 3.1, we obtain just one case for which Noether point symmetries exist. This is when $f(t)=\delta e^{-b t}, g(t)=\lambda e^{b t}$, where $\delta, b$, and $\lambda$ are constants. try

This case provides us with a single Noether point symme-

$$
\Gamma=\frac{\partial}{\partial t}-b u \frac{\partial}{\partial u}+b v \frac{\partial}{\partial v} \quad \text { with } B=0 .
$$

The Noetherian first integral associated with $\Gamma$ is

$$
I=\dot{u} \dot{v}+b(u \dot{v}-\dot{u} v)+\ln u v+\beta\left(\delta v e^{-b t}+\lambda u e^{b t}\right) .
$$

3.3. $p \neq-1, q=-1$. In this case the coupled inhomogeneous Lane-Emden system (5) becomes

$$
\begin{aligned}
& u^{\prime \prime}(t)+v^{p}(t)+\beta f(t)=0, \\
& v^{\prime \prime}(t)+\frac{1}{u(t)}+\beta g(t)=0,
\end{aligned}
$$

which has a first-order Lagrangian

$$
L=\dot{u} \dot{v}-\frac{1}{p+1} v^{p+1}-\ln u-\beta(v f(t)+u g(t)) .
$$

Carrying out similar procedure as above, we obtain the following one case for which Noether point symmetries exist. This is $f(t)=\delta, g(t)=\lambda$, where $\delta$ and $\lambda$ are constants.

This case provides us with one Noether point symmetry, namely,

$$
\Gamma=\frac{\partial}{\partial t} \quad \text { with } B=0 .
$$

The Noetherian first integral corresponding to $\Gamma$ is

$$
I=\dot{u} \dot{v}+\frac{v^{p+1}}{p+1}+\ln u+\beta(\delta v+\lambda u)
$$

3.4. $p=-1, q \neq-1$. In this case the coupled inhomogeneous Lane-Emden system (5) becomes

$$
\begin{aligned}
& u^{\prime \prime}(t)+\frac{1}{v(t)}+\beta f(t)=0, \\
& v^{\prime \prime}(t)+u^{q}(t)+\beta g(t)=0,
\end{aligned}
$$


for which a Lagrangian $L(t, u, v, \dot{u}, \dot{v})$ is given by

$$
L=\dot{u} \dot{v}-\ln v-\frac{1}{q+1} u^{q+1}-\beta(v f(t)+u g(t)) .
$$

Following the above procedure we obtain one case for which Noether point symmetries exist. This is $f(t)=\delta, g(t)=\lambda$, where $\delta$ and $\lambda$ are constants.

Here we obtain one Noether point symmetry, namely,

$$
\Gamma=\frac{\partial}{\partial t} \quad \text { with } B=0
$$

and the Noetherian first integral corresponding to $\Gamma$ is

$$
I=\dot{u} \dot{v}+\frac{u^{q+1}}{q+1}+\ln v+\beta(\delta v+\lambda u) .
$$

\section{Concluding Remarks}

In this paper we studied the coupled inhomogeneous LaneEmden system (5). Sufficient and necessary conditions for the existence of multiple positive solutions for this system were obtained in [12]. Here we performed the complete Noether symmetry classification of the system (5) with respect to a first-order Lagrangian. We considered four cases for different values of $p$ and $q$ separately and obtained several cases for the functions $f(t)$ and $g(t)$ which resulted in Noether point symmetries. For each of these cases we then presented the Noetherian first integral for the corresponding Noether operator. The importance of first integrals, particularly of systems, was mentioned in the Introduction section.

\section{References}

[1] H. Zou, "A priori estimates for a semilinear elliptic system without variational structure and their applications," Mathematische Annalen, vol. 323, no. 4, pp. 713-735, 2002.

[2] D. Qiuyi, "Entire positive solutions for inhomogeneous semilinear elliptic systems," Glasgow Mathematical Journal, vol. 47, no. 1, pp. 97-114, 2005.

[3] P. Clément, D. G. de Figueiredo, and E. Mitidieri, "Positive solutions of semilinear elliptic systems," Communications in Partial Differential Equations, vol. 17, no. 5-6, pp. 923-940, 1992.

[4] C. Cosner, "Positive solutions for superlinear elliptic systems without variational structure," Nonlinear Analysis: Theory, Methods \& Applications, vol. 8, no. 12, pp. 1427-1436, 1984.

[5] D. G. de Figueiredo and P. L. Felmer, "A Liouville-type theorem for elliptic systems," Annali della Scuola Normale Superiore di Pisa, vol. 21, no. 3, pp. 387-397, 1994.

[6] E. Mitidieri, "A Rellich type identity and applications," Communications in Partial Differential Equations, vol. 18, no. 1-2, pp. 125-151, 1993.

[7] E. Mitidieri, "Non-existence of positive solutions of semilinear elliptic systems in Rn," Differential and Integral Equations, vol. 9, pp. 456-479, 1996.

[8] L. A. Peletier and R. C. A. M. van der Vorst, "Existence and nonexistence of positive solutions of nonlinear elliptic systems and the biharmonic equation," Differential and Integral Equations, vol. 5, no. 4, pp. 747-767, 1992.
[9] W. Reichel and H. Zou, "Non-existence results for semilinear cooperative elliptic systems via moving spheres," Journal of Differential Equations, vol. 161, no. 1, pp. 219-243, 2000.

[10] R. C. A. M. van der Vorst, "Variational identities and applications to differential systems," Archive for Rational Mechanics and Analysis, vol. 116, no. 4, pp. 375-398, 1992.

[11] P. Han and Z. Liu, "Multiple positive solutions of strongly indefinite systems with critical Sobolev exponents and data that change sign," Nonlinear Analysis: Theory, Methods \& Applications, vol. 58, no. 1-2, pp. 229-243, 2004.

[12] D. Qiuyi and C. C. Tisdell, "Nondegeneracy of positive solutions to homogeneous second-order differential systems and its applications," Acta Mathematica Scientia B, vol. 29, no. 2, pp. 435-446, 2009.

[13] H. Goldstein, C. Poole, and J. Safko, Classical Mechanics, Pearson Education, Singapore, 3rd edition, 2004.

[14] V. M. Gorringe and P. G. L. Leach, "Lie point symmetries for systems of second order linear ordinary differential equations," Quaestiones Mathematicae, vol. 11, no. 1, pp. 95-117, 1988.

[15] B. Muatjetjeja and C. M. Khalique, "First integrals for a generalized coupled Lane-Emden system," Nonlinear Analysis: Real World Applications, vol. 12, no. 2, pp. 1202-1212, 2011.

[16] B. van Brunt, The Calculus of Variations, Universitext, Springer, New York, NY, USA, 2004.

[17] E. Noether, "Invariante Variationsprobleme, Königliche Gesellschaft der Wissenschaften Zu Göttingen, Nachrichten," Mathematisch-Physikalische Klasse Heft, vol. 2, pp. 235-269, 1918. 


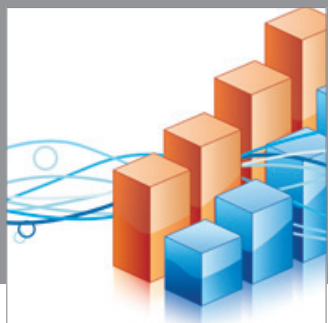

Advances in

Operations Research

mansans

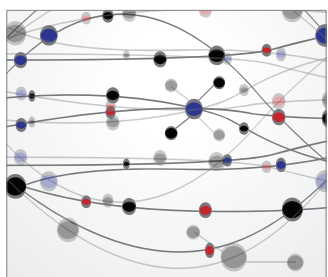

The Scientific World Journal
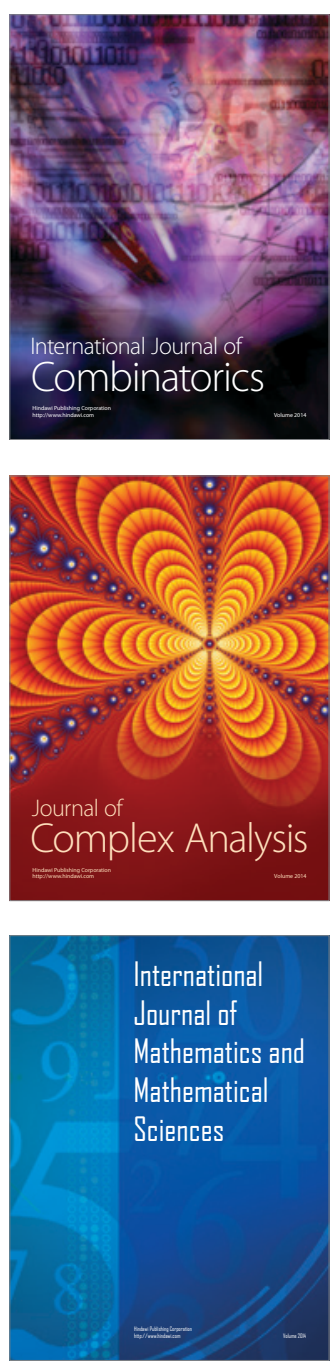
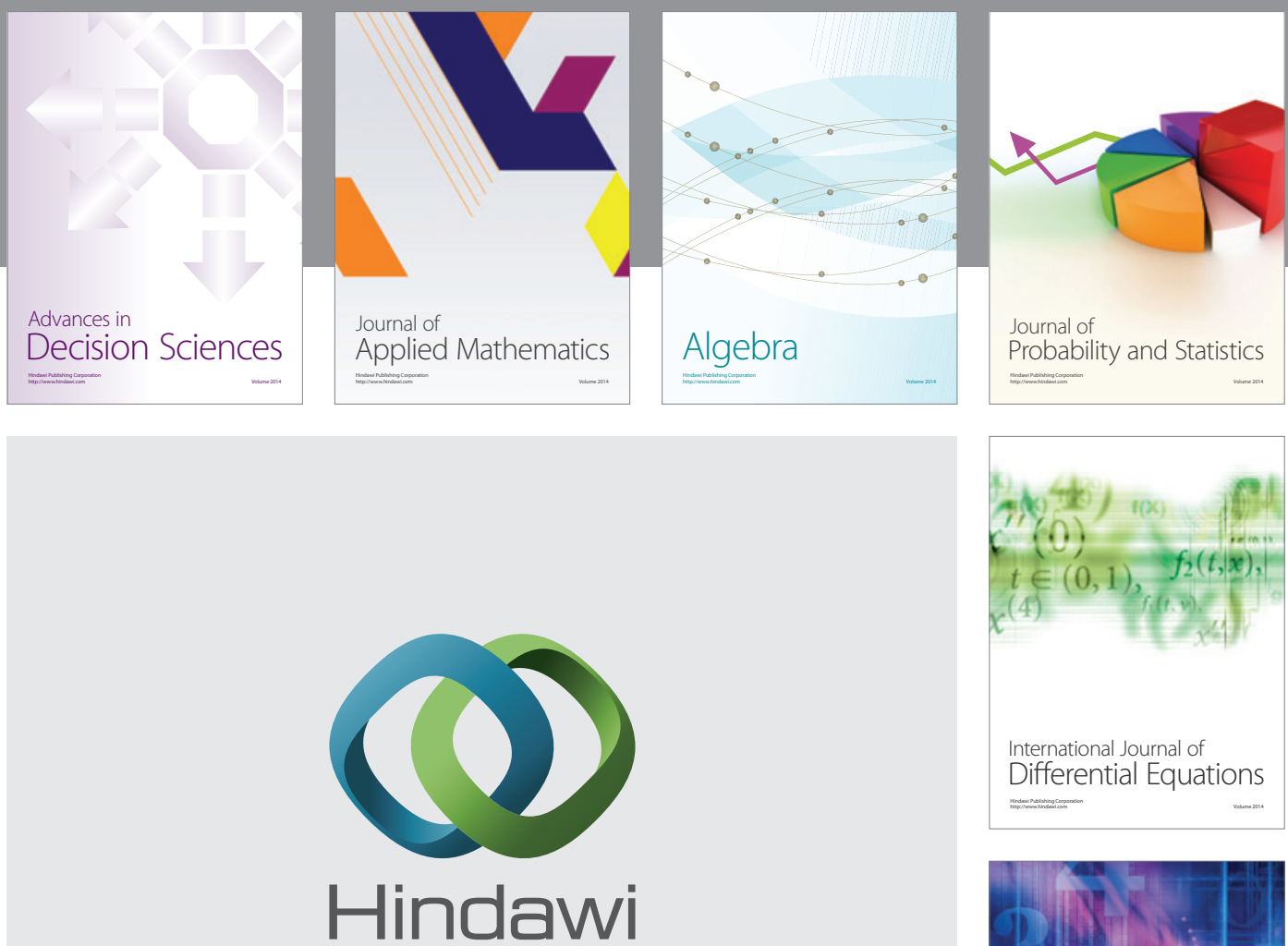

Submit your manuscripts at http://www.hindawi.com
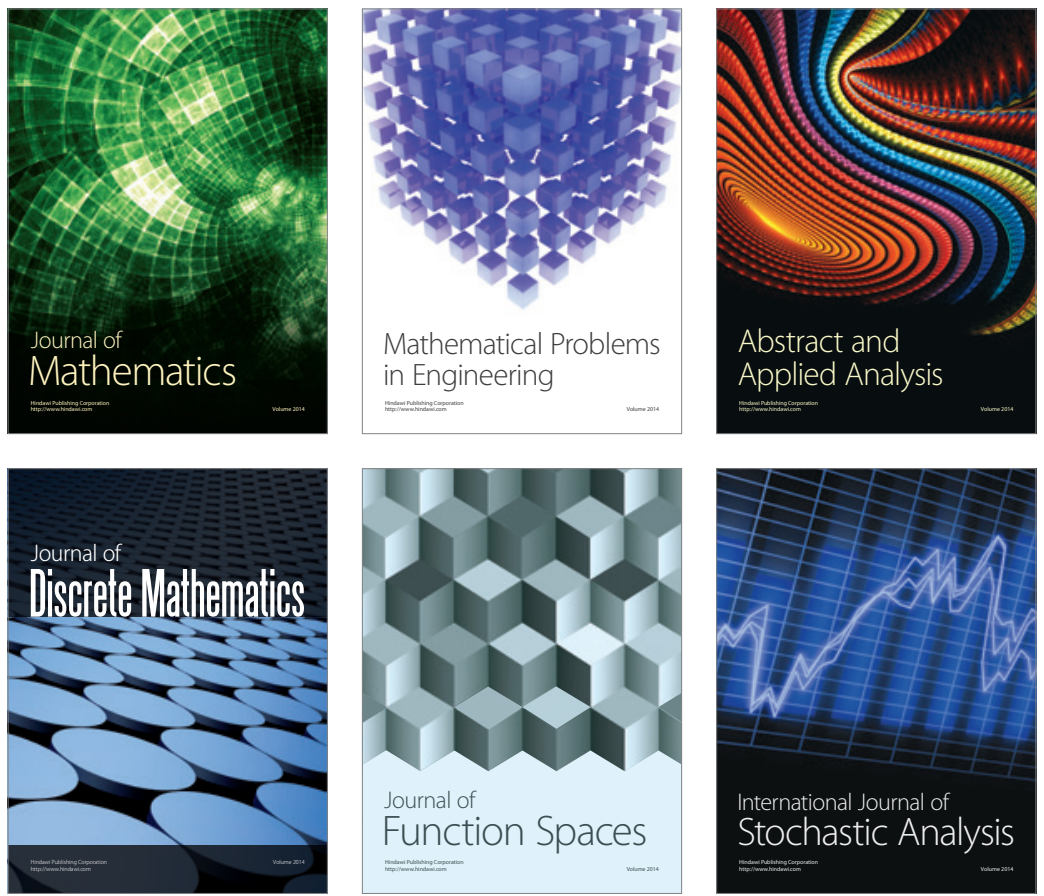

Journal of

Function Spaces

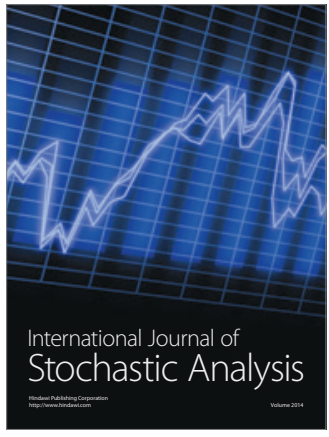

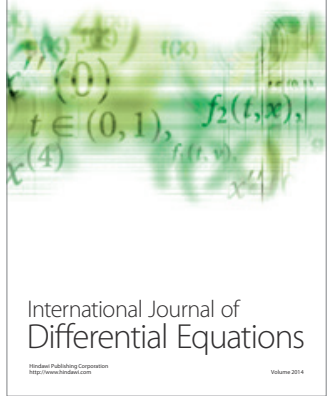
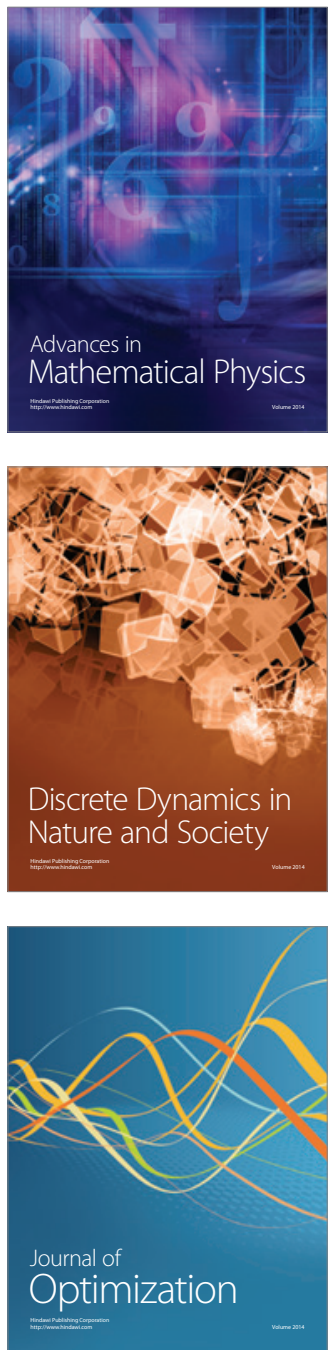